\title{
AN APPLICATION OF LOW-ORDER ARMA AND GARCH MODELS FOR SEA LEVEL FLUCTUATIONS
}

\author{
Tomasz Niedzielski ${ }^{a, b, 1}$, Wiesław Kosek ${ }^{a, c}$ \\ ${ }^{a}$ Space Research Centre, Polish Academy of Sciences, Poland \\ e-mails: niedzielski@cbk.waw.pl, kosek@cbk.waw.pl \\ ${ }^{b}$ Institute of Geography and Regional Development, University of Wrocław, Poland \\ ${ }^{c}$ Environmental Engineering and Land Surveying, University of Agriculture in Kraków, Poland
}

\begin{abstract}
The paper presents the analysis of geographically-dependent irregular sea level fluctuations, often referred to as residual terms around deterministic signals, carried out by means of stochastic low-order autoregressive moving average (ARMA) and generalised autoregressive conditional heteroscedastic (GARCH) models. The gridded sea level anomaly (SLA) time series from TOPEX/Poseidon (T/P) and Jason-1 (J-1) satellite altimetry, commencing on 10th January 1993 and finishing on 14th July 2003, has been examined. The aforementioned models, limited to low-orders being combinations of 0,1 and 2, have been fitted to the SLA data. The root mean square and the Shapiro-Wilk test for the normal distribution have been used to calculate statistics of the residuals from these models. It has been found that autoregressive (AR) models as well as ARMA ones serve well the purpose of adequate modelling irregular sea level fluctuations, with a successful fit in some patchy bits of the equatorial Pacific. In contrast, GARCH models have been shown to be rather inaccurate, specifically in the vicinity of the tropical Pacific, in the North Pacific and in the equatorial Indian Ocean. The pattern of the Tropical Instability Waves (TIWs) has been noticed in the statistics of AR and ARMA model residuals indicating that the dynamics of these waves cannot be captured by the aforementioned linear stochastic processes.
\end{abstract}

Keywords: Sea level modelling, Tropical Instability Waves, TOPEX/Poseidon, Jason-1.

\section{INTRODUCTION}

It is now well known that sea level changes vary depending on geographic location and time. There are numerous analyses targeting at both spatial and temporal description of sea level changes. In particular, such investigations show how various features of sea level temporal variability, either regular trends and oscillations or irregular stochastic fluctuations, depend on geographic location. Although this body of knowledge is rather complete, its application towards better modelling and prediction is still a challenging research activity.

\footnotetext{
${ }^{1}$ Currently at Oceanlab, University of Aberdeen, UK
} 
Trends in sea level time series are usually equated with rates calculated by fitting linear models. However, recent investigations indicate a certain level of nonlinearity in long-term trend components. The commonly cited estimates of the global mean sea level rise inferred from tide gauge measurements from 19th and 20th centuries are shown to be 1.0-2.0 mm/year (e.g. Douglas, 1991; Miller and Douglas, 2004), and departures from linear trends due to temporarily non-zero accelerations introduce various subtrends with rates from -1.5 to $3.5 \mathrm{~mm} /$ year between 1860 and 2000 (Jevrejeva et al., 2006). The satellite altimetry serves well the purpose of absolute sea level observations and, along with corrections for the glacial isostatic adjustment, helps to improve the accuracy of monitoring of dynamic ocean topography. The global mean sea level rise estimated from 1990's until 2000's by many authors varies between 1.5 and $3.4 \mathrm{~mm} /$ year (e.g. Kosek, 2001; Leuliette et al., 2004; Beckley et al., 2007; Cazenave et al., 2008; Ablain et al., 2009). Such estimates of a current trend from satellite altimetry have been also shown to vary with geographic locations, with rates ranging from -20 to $20 \mathrm{~mm}$ /year (Kosek, 2001; Cazenave et al., 2008).

Seasonal oscillations in sea level time series exhibit a wide range of periods and are driven by various geophysical processes, often linked to atmospheric and oceanic phenomena. The most meaningful ones in the global mean sea level change data are annual, semiannual, 120-days, 60-days and even shorter period oscillations. Similar components can be found at most geographic locations, however their amplitudes vary significantly and correspond to regional atmospheric and oceanic conditions (Kosek, 2001).

The overwhelmingly irregular changes in sea level variation are those which do not fall into the aforementioned categories. They are driven by some irregular climatic and oceanic phenomena, such as for instance the El Niño/Southern Oscillation (ENSO), the Pacific Decadal Oscillation (PDO) or the North Atlantic Oscillation (NAO). In terms of mathematical description, such irregularities are controlled by certain stochastic processes, the structure of which is not easy to recognise. There have been numerous studies towards a better recognition of such processes. Niedzielski and Kosek (2010) have recently offered an extensive analysis of the sea level anomaly (SLA) residuals from the TOPEX/Poseidon $(\mathrm{T} / \mathrm{P})$ and Jason-1 (J-1) altimetric satellites. At most locations SLA residuals follow the normal distribution, however there are a few regions where these fluctuations depart from the gaussian probability law. Some of them have been equated with the ENSO impact.

Many authors have carried out modelling and prediction of irregular signals in sea level variability. They included analyses of the global mean sea level time series (Niedzielski and Kosek, 2005; Iz, 2006) as well as regional site-specific data (Raudsepp et al., 1999; Barbosa et al., 2006, 2008; Niedzielski and Kosek, 2009). However, to date none of these exercises considers recommendations on a model structure which can be inferred from non-gaussian manner of variability. The purpose of the present study is to provide a preliminary assessment of how selected low-order autoregressive moving average (ARMA) and generalised autoregressive conditional heteroscedastic (GARCH) models describe the irregular SLA stochastic residuals.

\section{METHODS}

The stochastic SLA residuals, $Y_{t}=Y_{t}(\lambda, \varphi)$, have been calculated for each longitude $\lambda$ and latitude $\varphi$ using the following expression: 


$$
Y_{t}=Y_{t}(\lambda, \varphi)=X_{t}(\lambda, \varphi)-[\alpha(\lambda, \varphi) t+\beta(\lambda, \varphi)]-\sum_{i=1}^{3} A_{i}(\lambda, \varphi) \sin \left[\omega_{i} t+\phi_{i}(\lambda, \varphi)\right],
$$

where $X_{t}(\lambda, \varphi)$ is the input SLA time series; $\alpha(\lambda, \varphi)$ is a coefficient of linear function; $\beta(\lambda, \varphi)$ is an intercept of linear function; $A_{i}(\lambda, \varphi)$ are amplitudes; $\phi_{i}(\lambda, \varphi)$ are phases; and $\omega_{i}, i=1, \ldots, 3$, are frequencies corresponding to annual, semiannual and 62-days oscillations, respectively. The 62-days oscillation is an alias oscillation caused by inaccurate modelling of the M2 tide, especially in the costal regions, and is generated by measuring this tidal wave with the $\mathrm{T} / \mathrm{P}$ sampling frequency of 9.9156 cycles/day (Wagner et al., 1994; Katz et al., 1995).

Two specific classes of time series models have been applied. The first group comprises ARMA stochastic processes and the second class consists of GARCH stochastic models. The latter approach accounts for a nonlinear dynamics of variance, whereas ARMA techniques form typical linear stochastic processes.

A zero-mean ARMA process $Y_{t}$ of orders $p$ and $q$, commonly known as $\operatorname{ARMA}(p, q)$, is stationary and is given by the formula:

$$
Y_{t}-\sum_{i=1}^{p} a_{i} Y_{t-i}=Z_{t}+\sum_{j=1}^{q} b_{j} Z_{t-j}
$$

where $a_{i}, i=1, \ldots, p$, are autoregressive (AR) coefficients; $b_{j}, j=1, \ldots, q$, are moving average (MA) coefficients; $Z_{t}$ is a white noise time series with mean 0 and variance $\sigma^{2}$.

Residuals $R_{t}^{\text {ARMA }}$ from the fitted ARMA model $\widehat{Y}_{t}^{\text {ARMA }}$ are defined as:

$$
R_{t}^{\mathrm{ARMA}}=Y_{t}-\widehat{Y}_{t}^{\mathrm{ARMA}}
$$

A goodness-of-fit can be additionally estimated by the root mean square (RMS) given by the following formula:

$$
\operatorname{RMS}=\sqrt{\frac{1}{n} \sum_{t=1}^{n}\left(R_{t}^{\mathrm{ARMA}}\right)^{2}}
$$

where $n$ is a sample size.

A GARCH process $Y_{t}$ of orders $p$ and $q$, often referred to as $\operatorname{GARCH}(p, q)$, is given by:

$$
Y_{t}=Z_{t} \sigma_{t}
$$

where $Z_{t}$ is a sequence of independent and identically distributed random variables (iid) with the standard gaussian distribution (mean 0 and variance 1 ) and additionally the following condition holds:

$$
\sigma_{t}^{2}=a_{0}+\sum_{i=1}^{q} a_{i} Y_{t-i}^{2}+\sum_{j=1}^{p} b_{j} \sigma_{t-j}^{2}
$$

where $a_{i}, i=0, \ldots, q$, and $b_{j}, j=1, \ldots, p$, correspond to coefficients of autoregressive conditional heteroscedastic $(\mathrm{ARCH})$ and $\mathrm{GARCH}$ parts, respectively. GARCH models 
enable modelling of non-stationary time series whenever there is a reason to believe that their variance is time-varying.

Following equation (5), residuals $R_{t}^{\mathrm{GARCH}}$ from the fitted GARCH model $\hat{\sigma}_{t}^{\mathrm{GARCH}}$ are defined as the following ratio:

$$
R_{t}^{\mathrm{GARCH}}=\frac{Y_{t}}{\hat{\sigma}_{t}^{\mathrm{GARCH}}} .
$$

If a GARCH model is properly fitted, such residuals should follow the iid structure with the standard normal distribution.

The normality hypothesis, both for $R_{t}^{\mathrm{ARMA}}$ and $R_{t}^{\mathrm{GARCH}}$, can be verified using the Shapiro-Wilk test (Shapiro and Wilk, 1965; Royston, 1995).

\section{DATA}

The gridded SLA time series from $\mathrm{T} / \mathrm{P}$ and J-1 satellite altimetry have been considered in the study. The data have been acquired courtesy of the Center for Space Research, University of Texas at Austin, USA. The spatial coverage is limited to $65^{\circ} \mathrm{S}-65^{\circ} \mathrm{N}$, however the meridional extent is unlimited. The spatial latitude-longitude resolution of the SLA fields is approximately equal to $1^{\circ} \times 1^{\circ}$. The gridded SLA time series span the time interval from 10th January 1993 to 14th July 2003, with J-1 data attached to the time series obtained by its ancestor $\mathrm{T} / \mathrm{P}$.

We have processed the gridded SLA time series from T/P cycles no. 12-364 and J-1 cycles no. 4-56, with missing data completed by means of linear interpolation. In order to construct the combined gridded SLA time series from both satellites the common observational interval (cycles no. 347-364 and 4-21, for $\mathrm{T} / \mathrm{P}$ and $\mathrm{J}-1$, respectively) has been taken into account (Fig. 1). For individual grids, relative offsets between both SLA time series, determined within the common observational interval, have been computed, averaged, and a resulting mean value has been added to the SLA data from J-1 to build the combined SLA time series for each grid point.

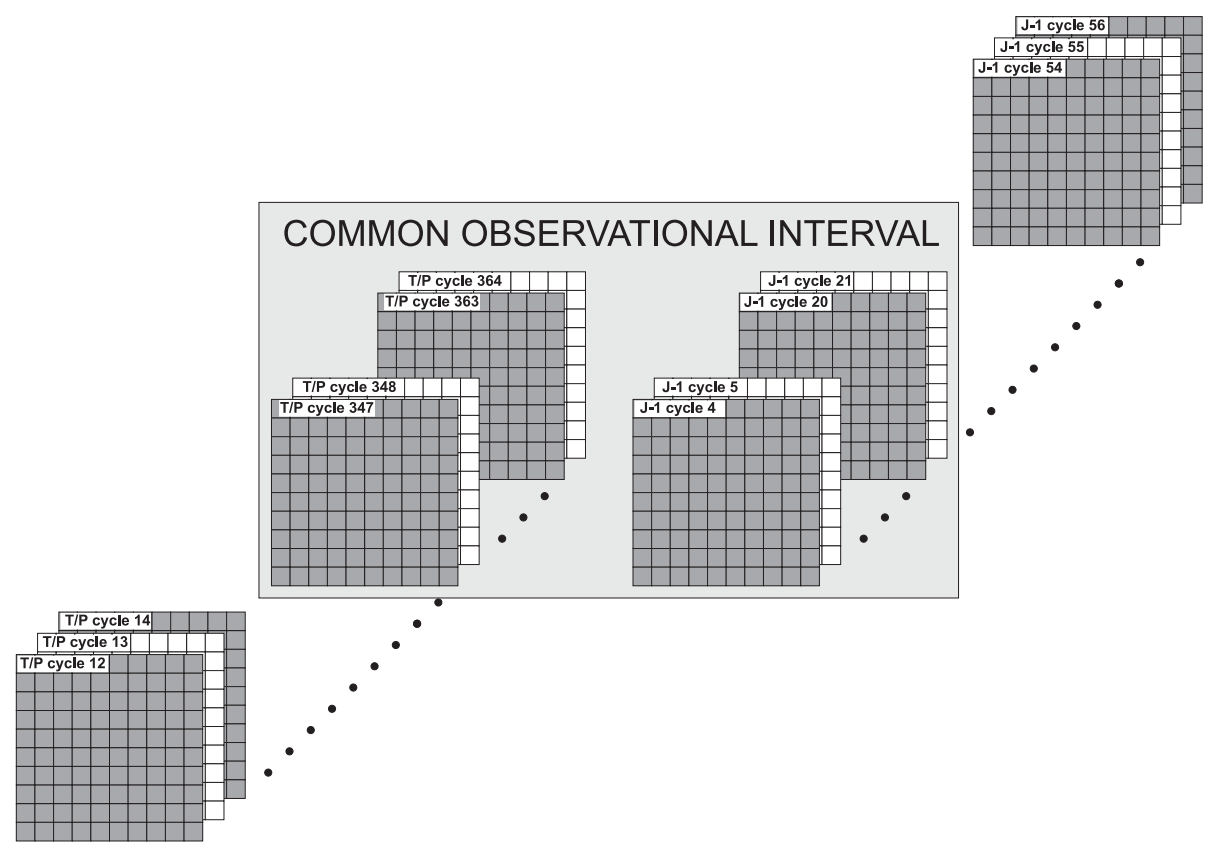

Fig. 1. Schematic view of a procedure used to merge T/P and J-1 gridded SLA data 


\section{RESULTS}

The first exercise compares performances of several ARMA models, i.e. $\operatorname{ARMA}(1,0)$, $\operatorname{ARMA}(0,1), \operatorname{ARMA}(1,1), \operatorname{ARMA}(2,0), \operatorname{ARMA}(0,2)$ and $\operatorname{ARMA}(2,2)$. Figs. 2 and 3 present the goodness-of-fit evaluated by means of RMS of model residuals. It has been inferred that $\mathrm{AR}$ models, i.e. $\operatorname{ARMA}(1,0)$ and $\operatorname{ARMA}(2,0)$, as well as $\operatorname{ARMA}$ ones, i.e. $\operatorname{ARMA}(1,1)$ and $\operatorname{ARMA}(2,2)$, provide the most accurate goodness-of-fit. In contrast, MA models, i.e. $\operatorname{ARMA}(0,1)$ and $\operatorname{ARMA}(0,2)$, have been shown to reveal worse performance. This has been additionally confirmed by basic statistics derived from the RMS estimates (Tab. 1).

Tab. 1. Basic RMS statistics of model residuals presented in Figs. 2 and 3

\begin{tabular}{|c|c|c|c|}
\hline Model & $\begin{array}{c}\text { Minimum RMS } \\
{[\mathrm{cm}]}\end{array}$ & $\begin{array}{c}\text { Maximum RMS } \\
{[\mathrm{cm}]}\end{array}$ & $\begin{array}{c}\text { Mean RMS } \\
{[\mathrm{cm}]}\end{array}$ \\
\hline ARMA(1,0) & 1.28 & 9.45 & 2.32 \\
\hline ARMA(0,1) & 1.37 & 9.39 & 2.84 \\
\hline ARMA(1,1) & 1.27 & 9.32 & 2.29 \\
\hline ARMA(2,0) & 1.27 & 9.24 & 2.30 \\
\hline ARMA(0,2) & 1.32 & 9.33 & 2.55 \\
\hline ARMA(2,2) & 1.25 & 9.08 & 2.27 \\
\hline
\end{tabular}

It has been noticed that for MA models the most significant and spatially large departures of models from the data are aligned along the Equator. This may be associated with ENSO dynamics as extensively discussed by Niedzielski and Kosek (2010). However, for AR and ARMA models such large-scale deviations have been shown to be gathered in the Southern Hemisphere.

The RMS of AR and ARMA model residuals very clearly show the presence of the Tropical Instability Waves (TIWs), known also as Legeckis waves, in the equatorial Pacific in the form of equidistant spots separated by $1100 \mathrm{~km}$ (Legeckis, 1977; Philander et al., 1986; Lawrence and Angell, 2000; Liu et al., 2000; Kosek, 2001; Pezzi et al., 2006) (Figs. 2 and 3). The TIW pattern seen in Figs. 2 and 3 commences around Galapagos in the eastern tropical Pacific, extends westward, and vanishes in the western equatorial Pacific. It has been inferred that AR and ARMA models fitted to irregular residuals cannot capture the dynamics of the TIWs.

The superior performance of AR and ARMA processes has also been inferred from the analysis of probability distributions of $R_{t}^{\mathrm{ARMA}}$ time series. Although a considerable gathering of non-gaussian SLA residuals from $\operatorname{ARMA}(1,0), \operatorname{ARMA}(2,0), \operatorname{ARMA}(1,1)$ and $\operatorname{ARMA}(2,2)$ models has been observed in the Pacific, the picture of normality rejection is spatially patchy and does not differ much from a corresponding view for the Atlantic Ocean (Figs. 4A, 4C, 5A, 5C). A different result has been found for MA processes, i.e. $\operatorname{ARMA}(0,1)$ and $\operatorname{ARMA}(0,2)$, which have been shown to produce inadequate models for the equatorial Pacific (Figs. 4B, 5B). This finding can be interpreted by the fact that MA processes lack the retrospective component of $Y_{t}$ and hence the past of the input residuals cannot be captured. 
(A) Residuals from fitted ARMA $(1,0)$ model $[\mathrm{cm}]$

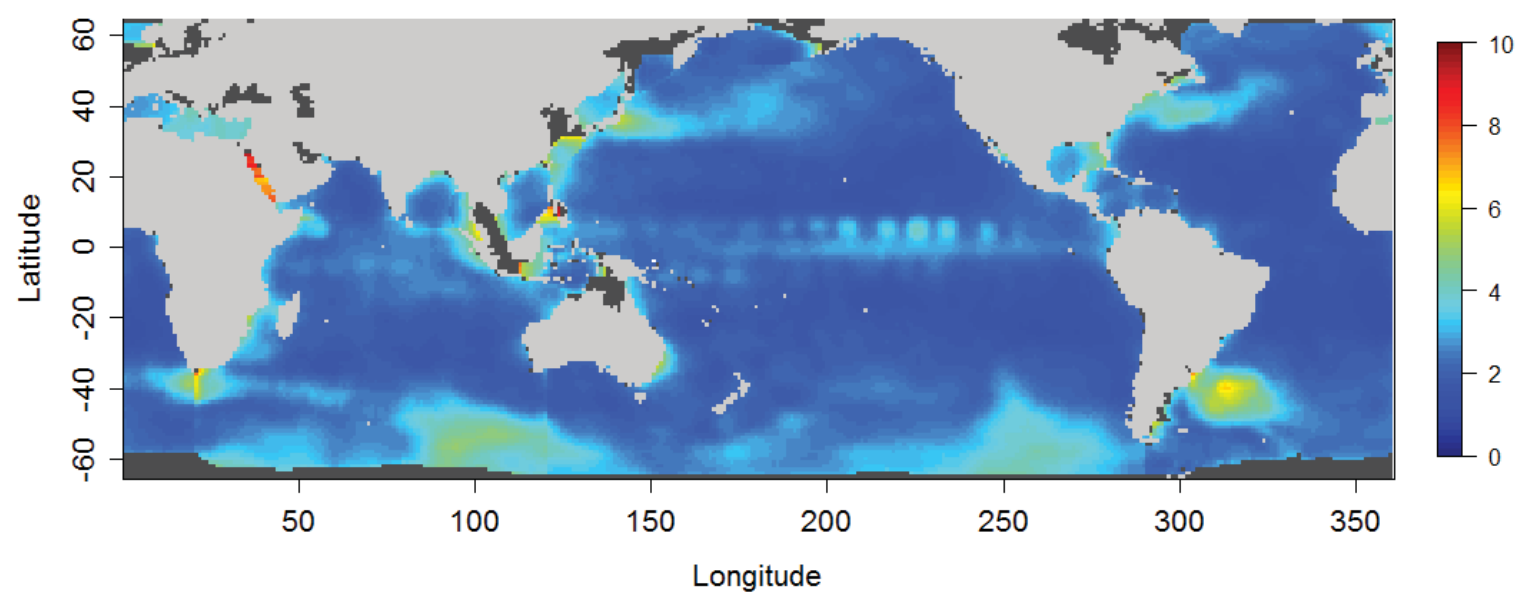

(B) Residuals from fitted ARMA $(0,1)$ model $[\mathrm{cm}]$

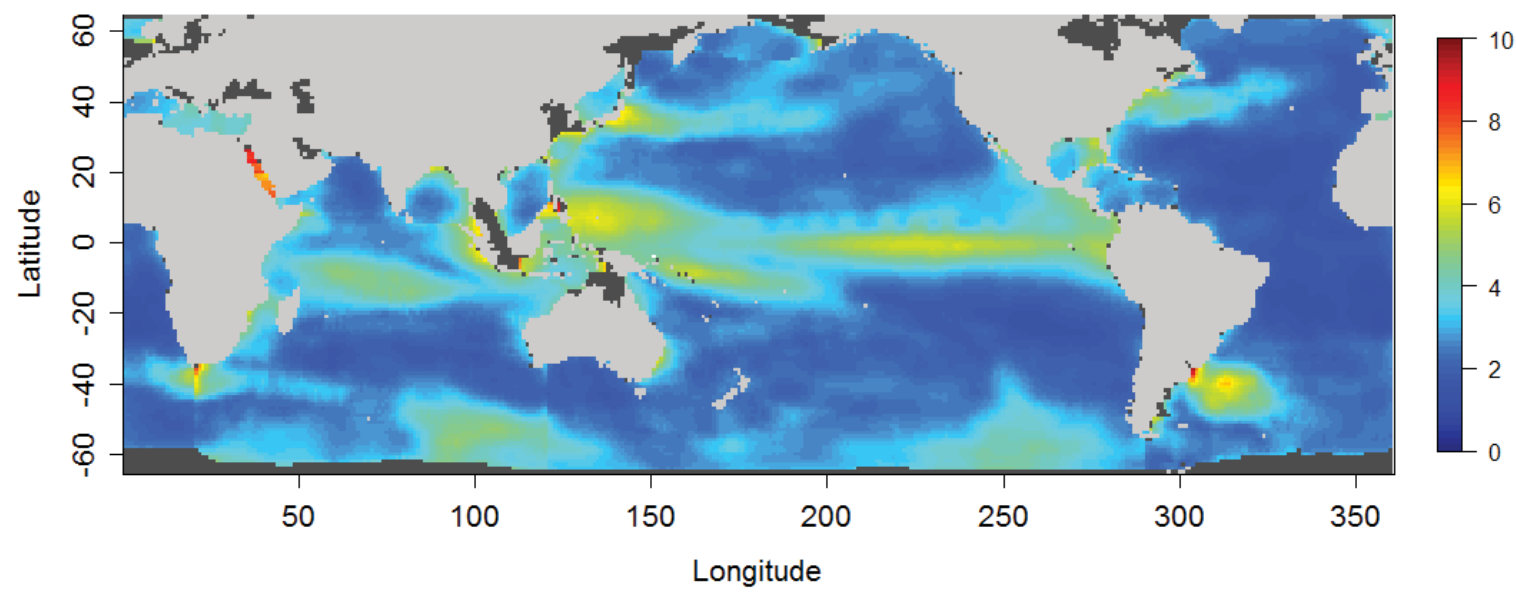

(C) Residuals from fitted ARMA $(1,1)$ model $[\mathrm{cm}]$

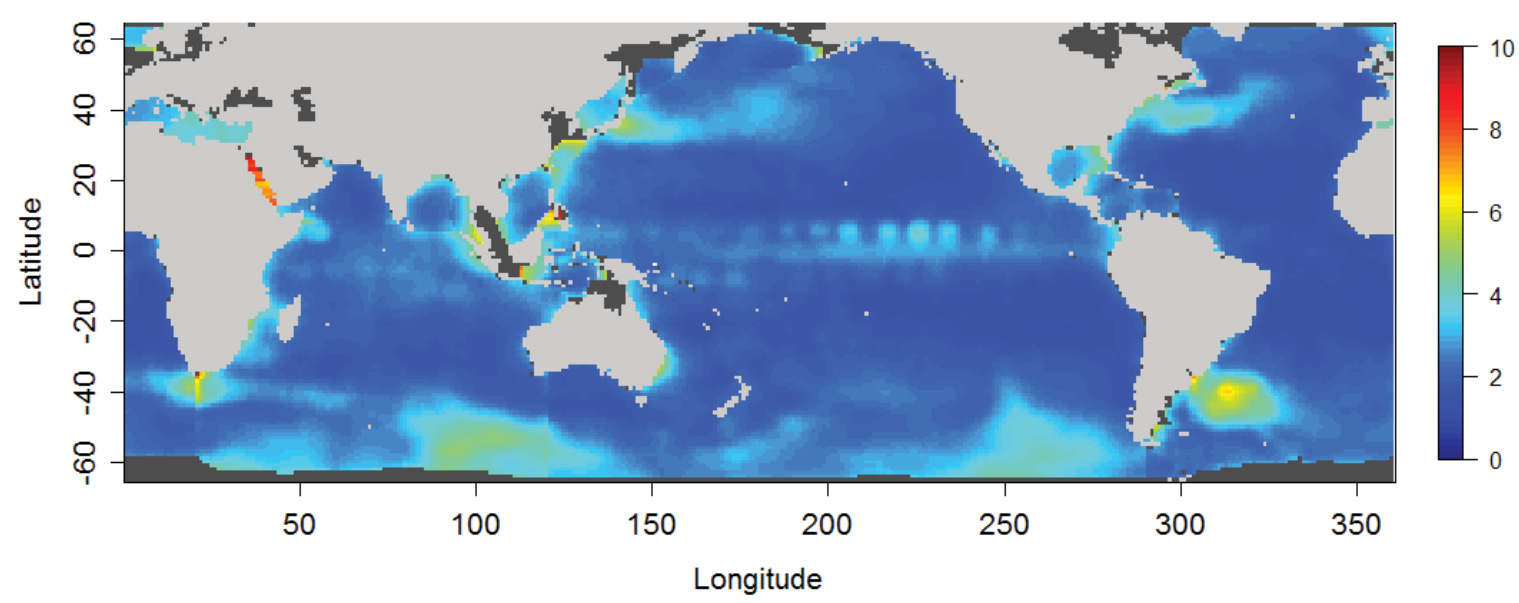

Fig. 2. RMS of model residuals for $\operatorname{ARMA}(1,0)(\mathrm{A}), \operatorname{ARMA}(0,1)(\mathrm{B}), \operatorname{ARMA}(1,1)(\mathrm{C})$; no data or inadequate interpolation grid points are marked in dark gray 
(A) Residuals from fitted ARMA $(2,0)$ model [cm]

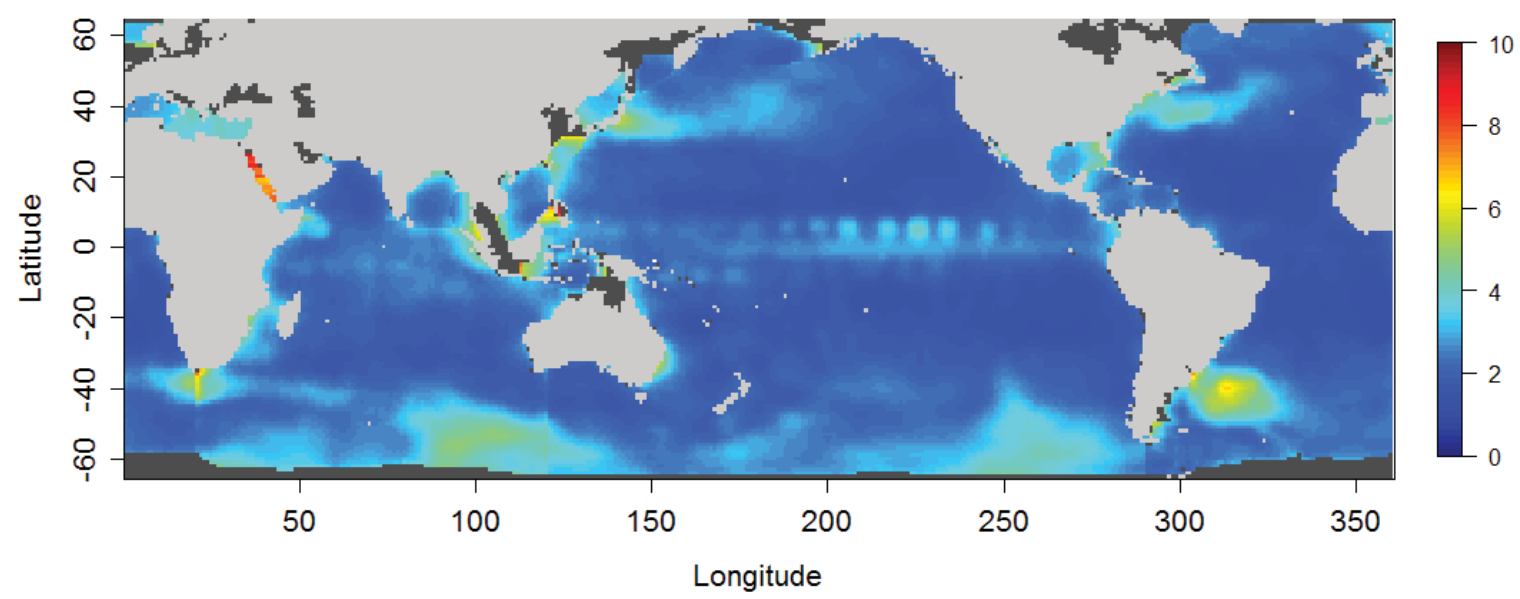

(B) Residuals from fitted ARMA $(0,2)$ model [cm]

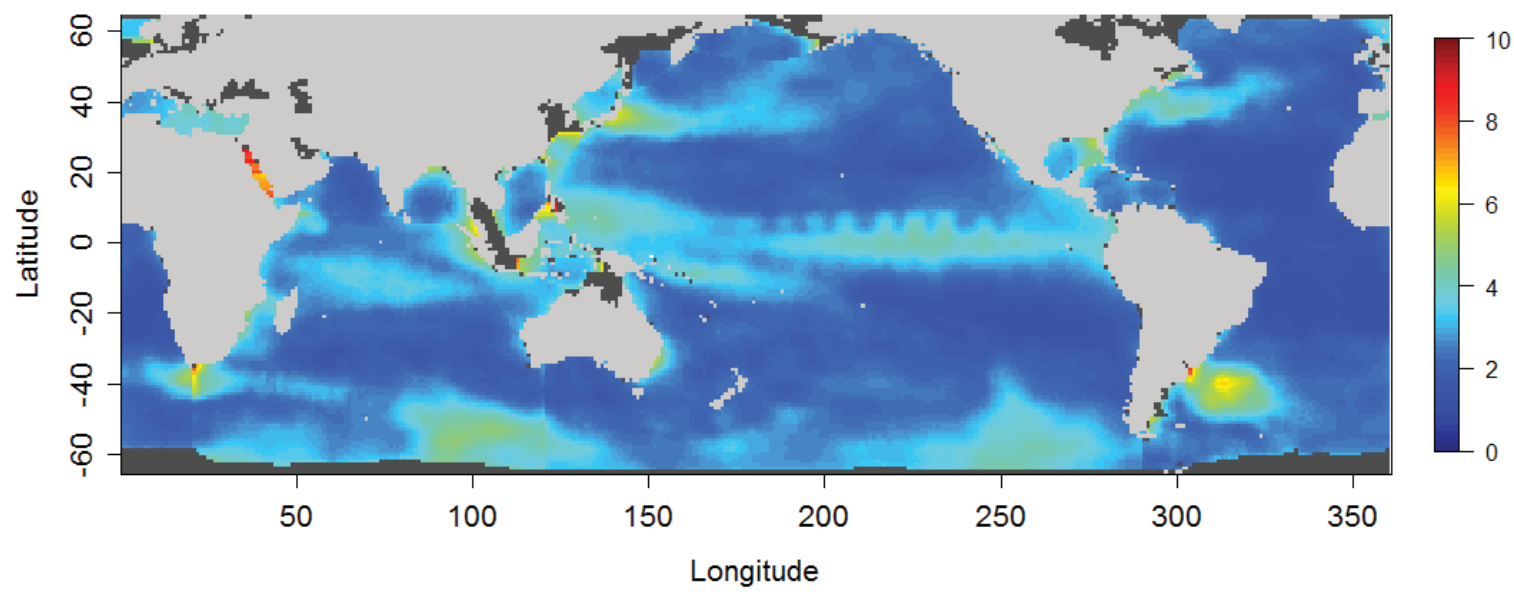

(C) Residuals from fitted ARMA(2,2) model [cm]

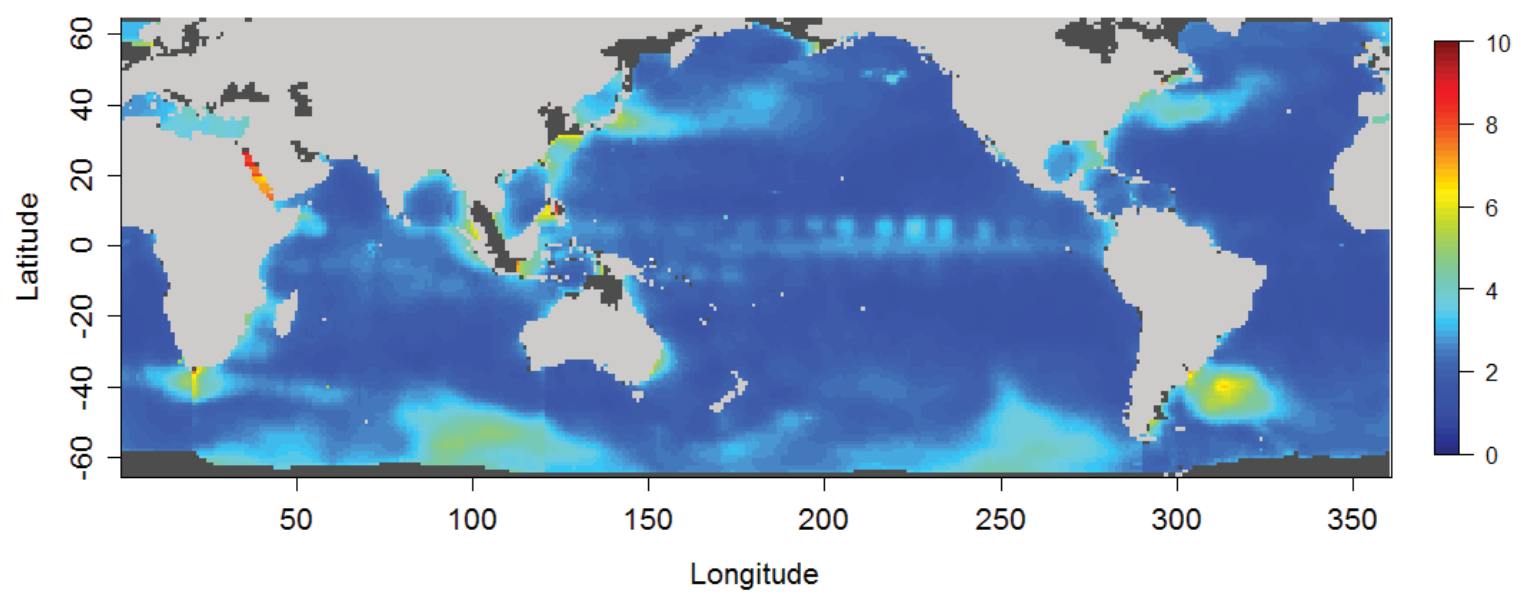

Fig. 3. RMS of model residuals for $\operatorname{ARMA}(2,0)(\mathrm{A}), \operatorname{ARMA}(0,2)(\mathrm{B}), \operatorname{ARMA}(2,2)(\mathrm{C})$; no data or inadequate interpolation grid points are marked in dark gray 
(A) P-value of Shapiro-Wilk test for residuals from fitted ARMA $(1,0)$ model

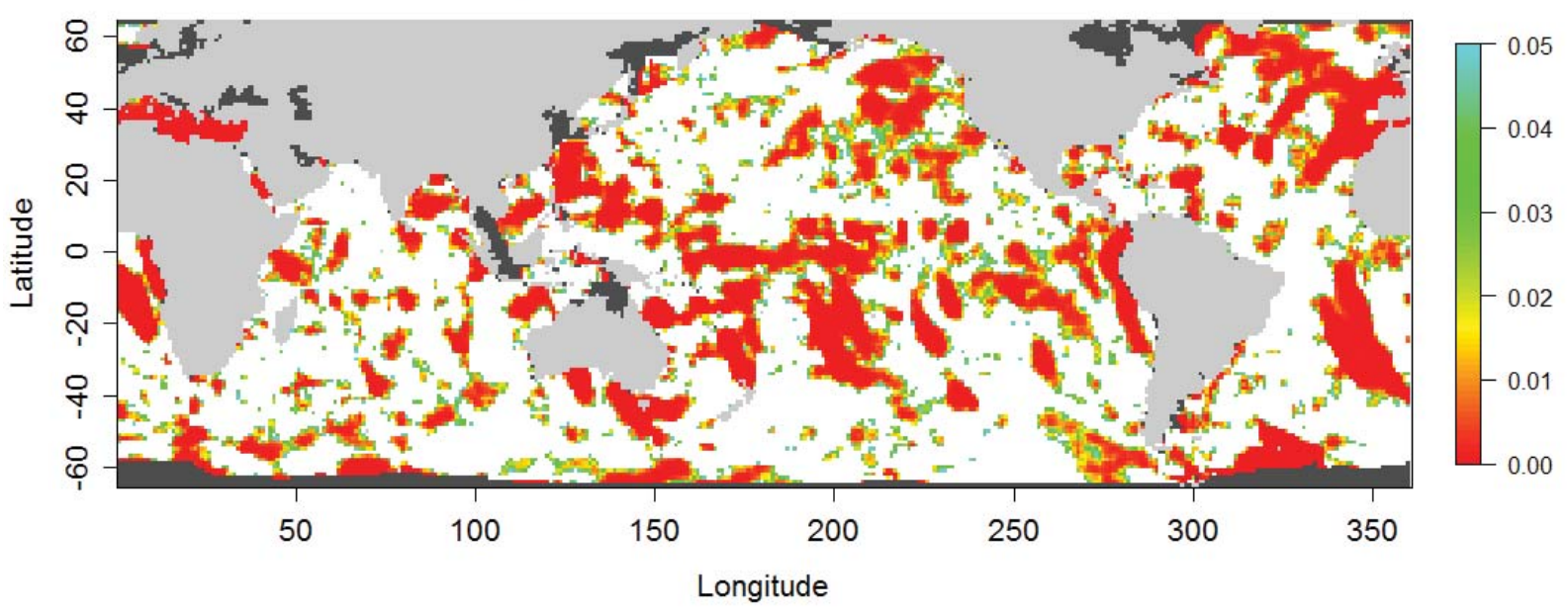

(B) P-value of Shapiro-Wilk test for residuals from fitted ARMA $(0,1)$ model

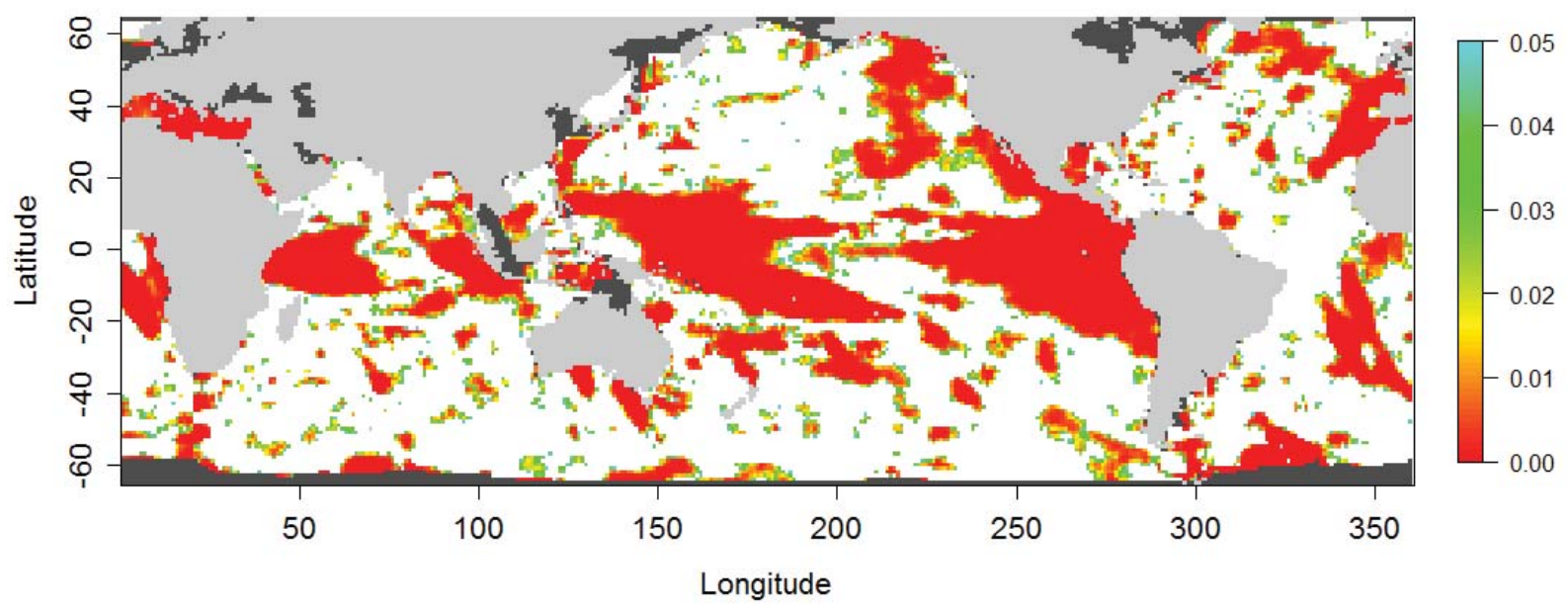

(C) P-value of Shapiro-Wilk test for residuals from fitted ARMA $(1,1)$ model

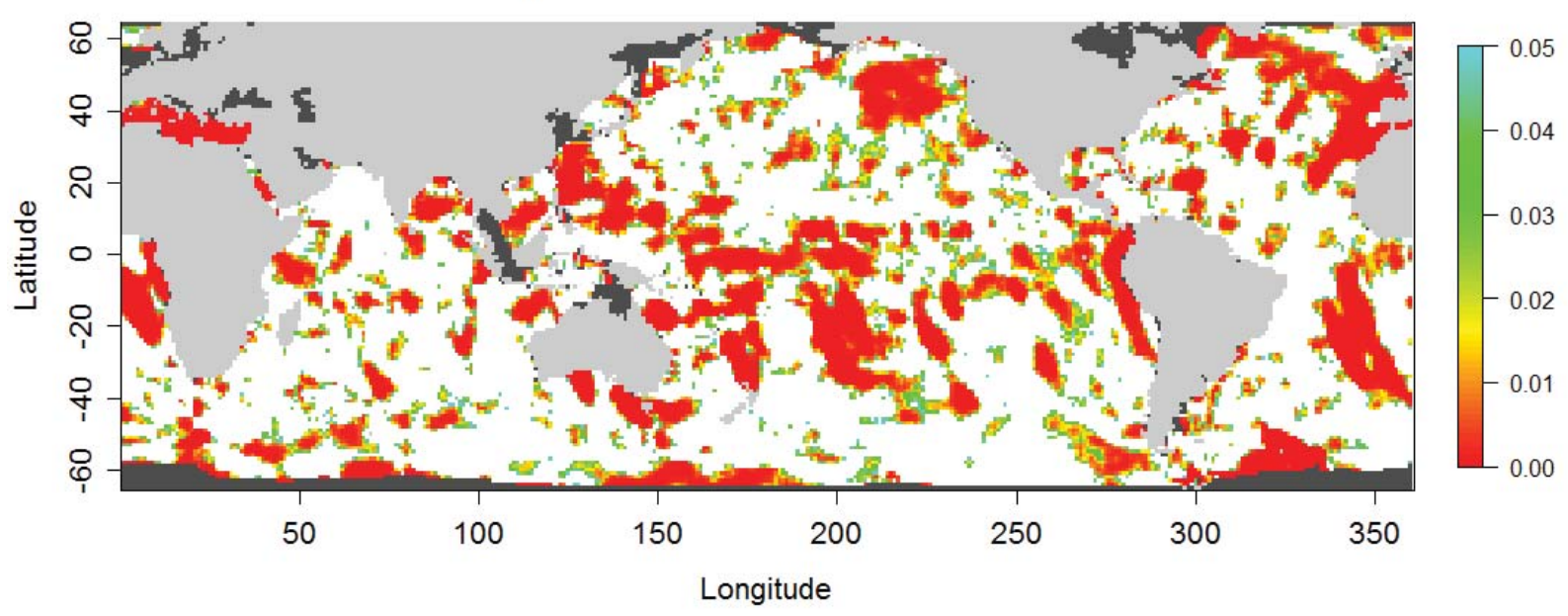

Fig. 4. P-values of Shapiro-Wilk test for $\operatorname{ARMA}(1,0)(\mathrm{A}), \operatorname{ARMA}(0,1)(B)$, and $\operatorname{ARMA}(1,1)(\mathrm{C})$; no data or inadequate interpolation grid points are marked in dark gray 
(A) P-value of Shapiro-Wilk test for residuals from fitted $\operatorname{ARMA}(2,0)$ model

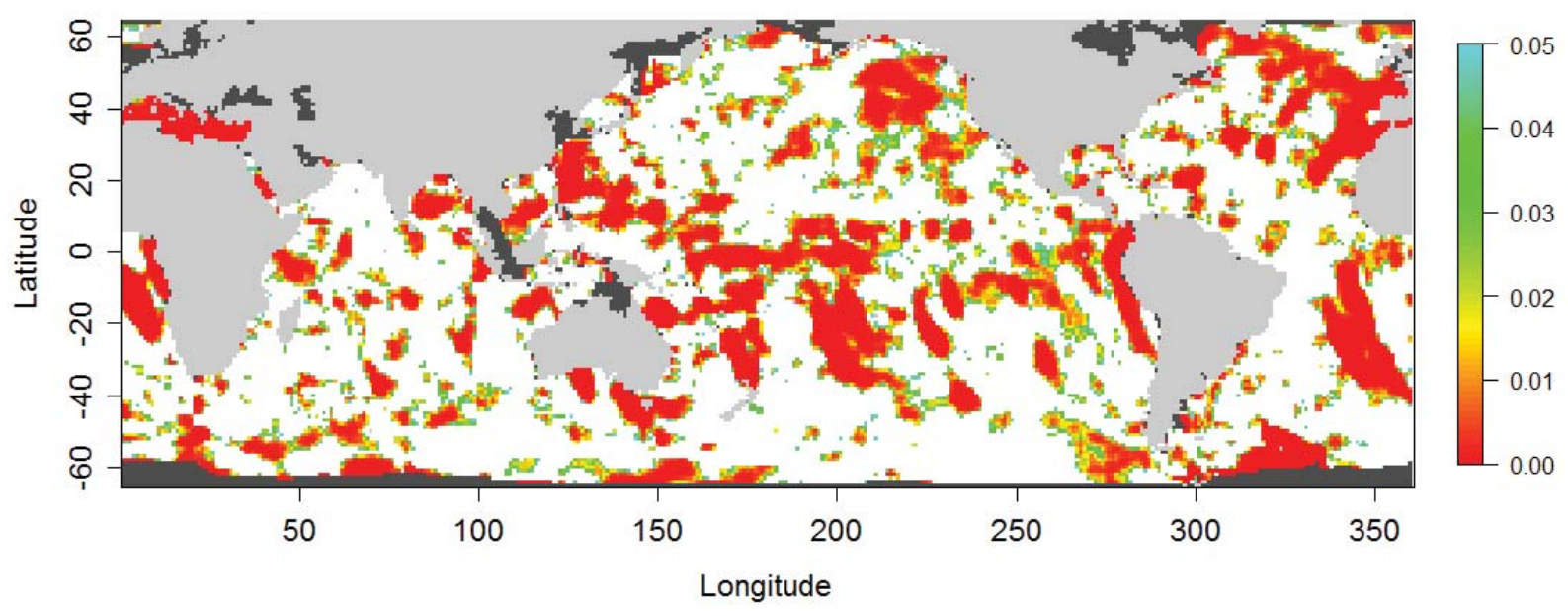

(B) P-value of Shapiro-Wilk test for residuals from fitted $\operatorname{ARMA}(0,2)$ model

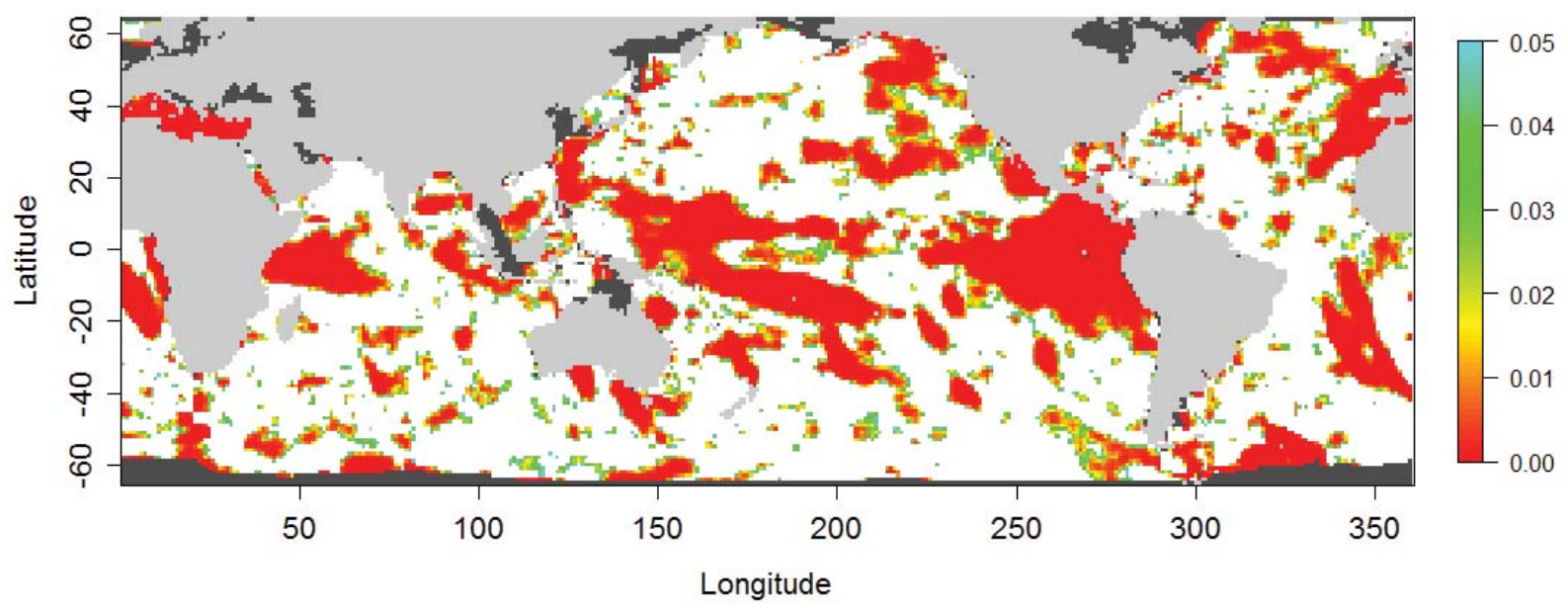

(C) P-value of Shapiro-Wilk test for residuals from fitted ARMA $(2,2)$ model

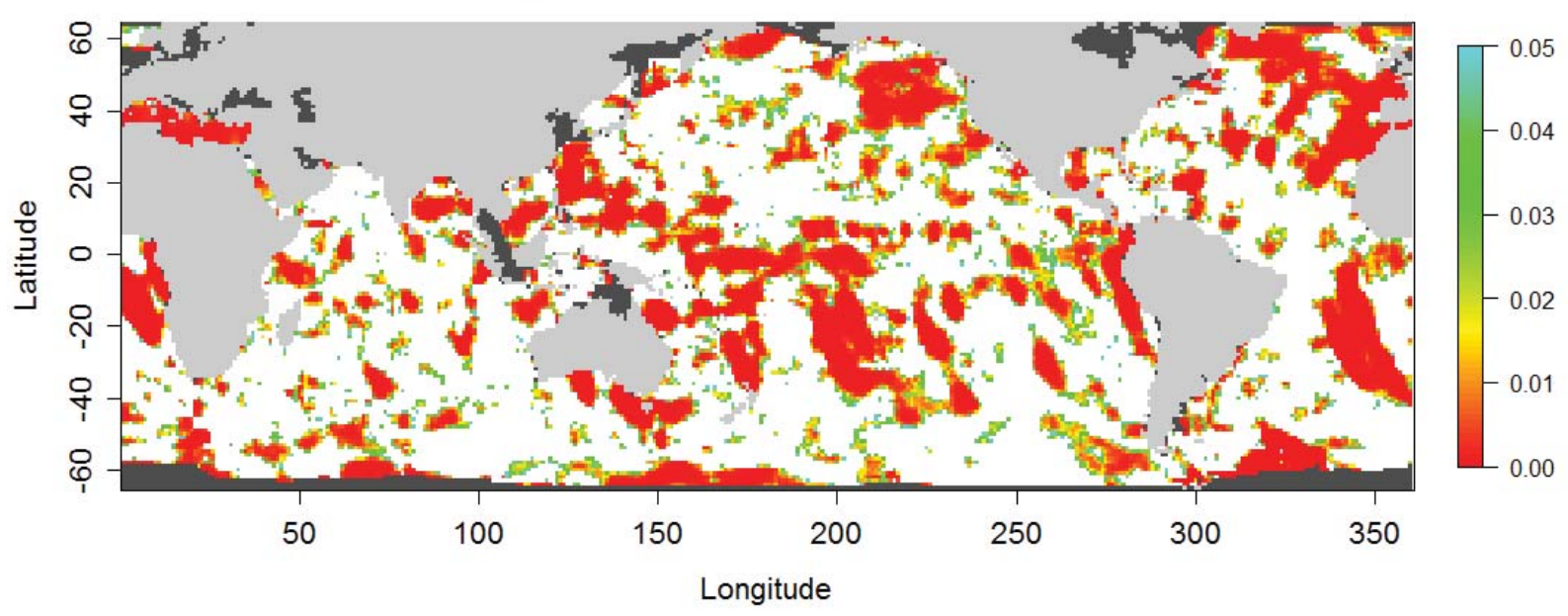

Fig. 5. P-values of Shapiro-Wilk test for $\operatorname{ARMA}(2,0)(\mathrm{A}), \operatorname{ARMA}(0,2)(\mathrm{B}), \operatorname{ARMA}(2,2)$ $(\mathrm{C})$; no data or inadequate interpolation grid points are marked in dark gray 
(A) P-value of Shapiro-Wilk test for residuals from fitted $\operatorname{GARCH}(1,0)$ model

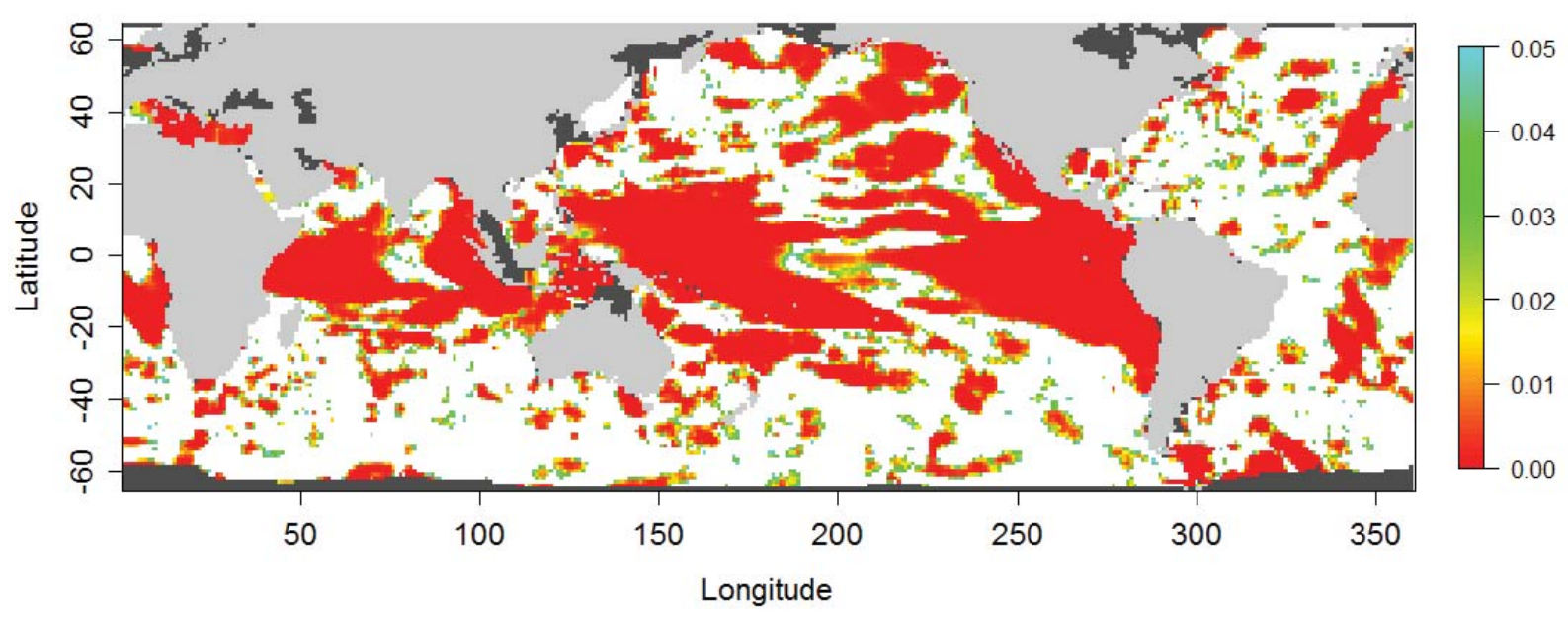

(B) P-value of Shapiro-Wilk test for residuals from fitted $\operatorname{GARCH}(0,1)$ model

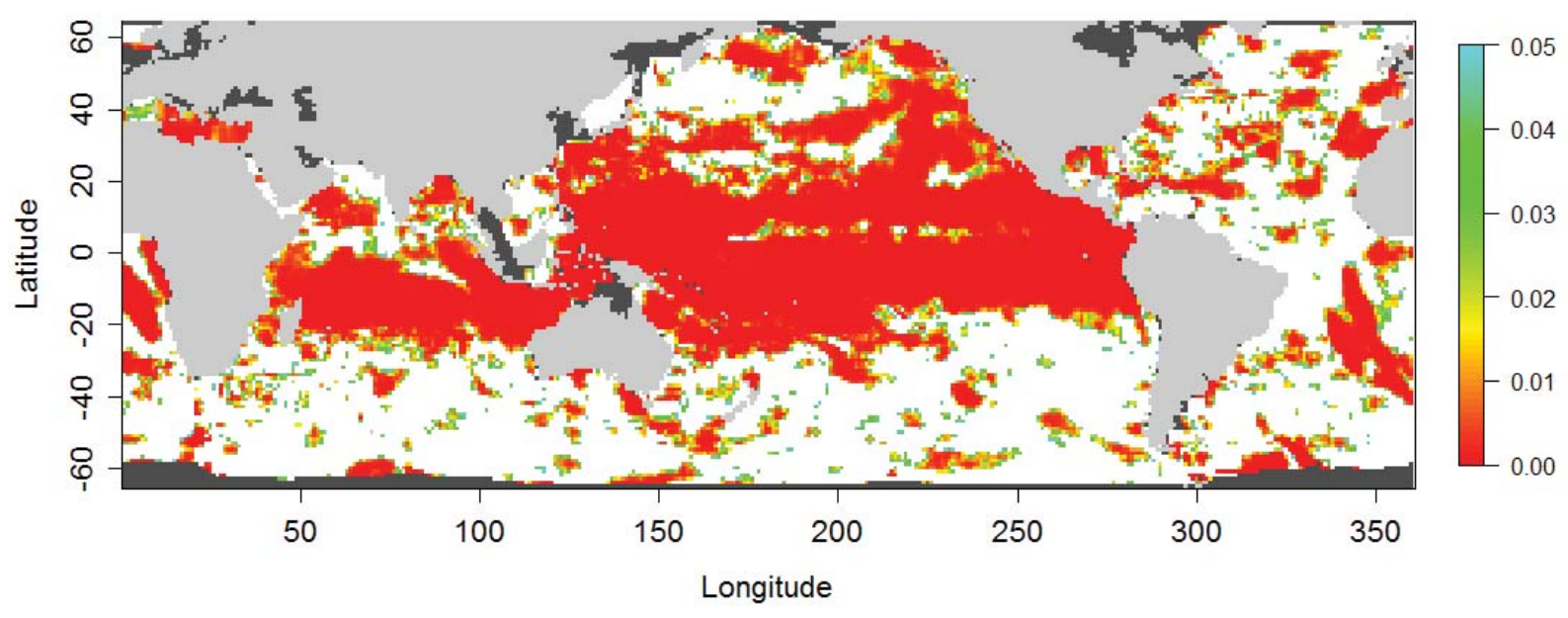

(C) P-value of Shapiro-Wilk test for residuals from fitted $\operatorname{GARCH}(1,1)$ model

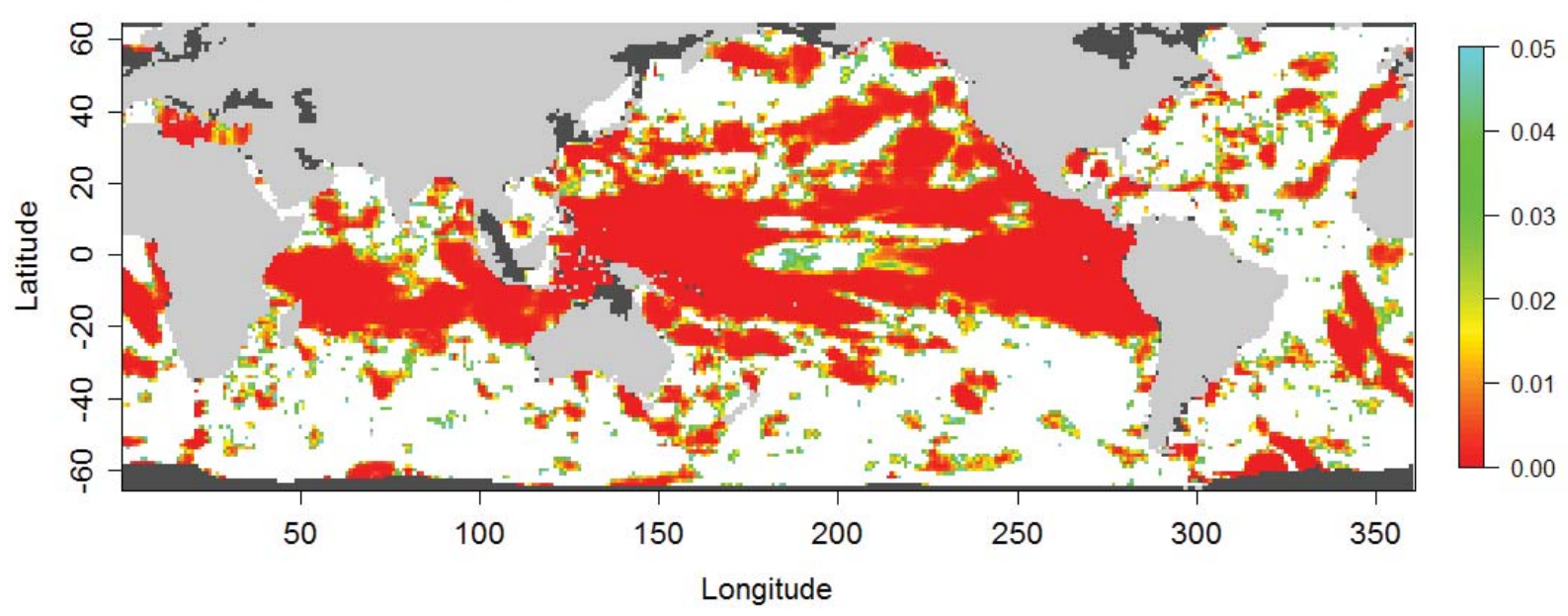

Fig. 6. P-values of Shapiro-Wilk test for $\operatorname{GARCH}(1,0)(\mathrm{A}), \operatorname{GARCH}(0,1)(\mathrm{B})$, $\operatorname{GARCH}(1,1)(\mathrm{C})$; no data or inadequate interpolation grid points are marked in dark gray 
The assessment of $R_{t}^{\mathrm{ARMA}}$, in terms of the RMS estimates and the Shapiro-Wilk test for normality, has shown that AR or ARMA processes can be quite well fitted to irregular SLA residuals at many geographic regions of the global ocean. However, these techniques cannot serve as general models for all locations because quite often more complex models should be recommended. Our study supplements the predictive analysis by Niedzielski and Kosek (2009) in the sense that it shows that low-order ARMA methods can have dissimilar performance in modelling irregular sea level fluctuations in the eastern tropical Pacific. This is also in agreement with what has been envisaged by Niedzielski (2010) who assumed linear processes to serve well the purpose of irregular sea level modelling at some sites located off the Equator in the eastern equatorial Pacific.

The second exercise highlighted in the present paper focuses on low-order GARCH modelling and aims at comparing $\operatorname{GARCH}(1,0), \operatorname{GARCH}(0,1)$ and $\operatorname{GARCH}(1,1)$ model residuals. The successful modelling of time-varying conditional variance needs to coincide with standard normality of $R_{t}^{\mathrm{GARCH}}$ residuals. The results of the Shapiro-Wilk test have shown that in large and spatially coherent ocean areas the probability distributions of $R_{t}^{\mathrm{GARCH}}$ residuals differ from the gaussian one (Fig. 6).

Note that the similar exercise has been performed by Niedzielski and Kosek (2010). Indeed, $p$-value of the Shapiro-Wilk test has been calculated for $Y_{t}$ residual SLA time series (Fig. 6; Niedzielski and Kosek, 2010), which is equivalent with the analysis of $Y_{t} / \hat{\sigma}$ time series with a constant standard deviation $\hat{\sigma}$. Unlike the latter case, in the present study the conditional time-varying standard deviation estimate $\hat{\sigma}_{t}$ has been used in the denominator. This allows to account for time-varying variance of the time series. However, it is apparent from Fig. 6 that the normality condition does not hold for most regions, particularly within a wide zone aligned along the Equator in the Pacific and Indian Ocean as well as in the North Pacific. Hence, taking into account a nonlinear variance structure has not been found to improve model performance.

In terms of a pure statistical evaluation of model residuals, low-order GARCH processes have been shown to be less adequate for modelling irregular SLA residuals than low-order ARMA processes. It is somewhat unexpected because ENSO-vulnerable regions, such as the equatorial Pacific, are characterised by time-varying variance which increases during El Niño and La Niña episodes. It does not necessarily mean that GARCH models are useless in such investigations. It is possible that higher-order GARCH processes can serve as tools for sea level change modelling.

\section{CONCLUSIONS}

The scope of the article covers modelling irregular gridded SLA time series from $\mathrm{T} / \mathrm{P}$ and J-1 satellite altimetry with selected low-order ARMA and GARCH models.

It has been found that AR and ARMA low-order models can be well fitted to irregular SLA residuals, however this finding holds for certain geographic locations including some bits of the equatorial Pacific. In contrast, MA low-order processes are not adequate for modelling irregular SLA residuals, neither in the western nor the eastern tropical Pacific. The possible interpretation is that MA models do not incorporate past values of the residual SLA data. The spatial pattern of the TIWs has been distinguished in the RMS of AR and ARMA model residuals. This finding shows that the dynamics of the Legeckis waves cannot be modelled by the applied linear stochastic processes. 
Surprisingly, GARCH low-order models do not serve well as adequate modelling techniques for irregular sea level fluctuations, particularly in the equatorial and North Pacific and in the tropical Indian Ocean. Although GARCH low-order processes offer time-varying variance modelling, they cannot be successfully applied in describing irregular sea level residuals.

Acknowledgments. The research has been financed from the Polish science funds by Polish Ministry of Science and Higher Education through the grant no. N N526 160136 under leadership of Dr Tomasz Niedzielski. The altimetric data have been acquired courtesy of the Center for Space Research, University of Texas at Austin, USA. We are also grateful to the authors of R 2.9.0 - A Language and Environment and additional packages.

\section{REFERENCES}

Ablain M., Cazenave A., Valladeau G., Guinehut S. (2009) A new assessment of the error budget of global mean sea level rate estimated by satellite altimetry over 1993-2008, Ocean Science, Vol. 5, 193-201.

Barbosa S.M., Silva M.E., Fernandes M.J. (2006) Multivariate autoregressive modelling of sea level time series from TOPEX/Poseidon satellite altimetry, Nonlinear Processes in Geophysics, Vol. 13, 177-184.

Barbosa S.M., Silva M.E., Fernandes M.J. (2008) Time Series Analysis of Sea-Level Records: Characterising Long-Term Variability, Nonlinear Time Series Analysis in the Geosciences, Lecture Notes in Earth Sciences, Vol. 112, 153-173

Beckley B.D., Lemoine F.G., Luthcke S.B., Ray R.D., Zelensky N.P. (2007) A reassessment of global rise and regional mean sea level trends from TOPEX/Poseidon and Jason-1 altimetry based on revised reference frame orbits, Geophysical Research Letters, Vol. 34, L14608, doi:10.1029/2007GL030002.

Cazenave A., Lombard A., Llovel W. (2008) Present-day sea level rise: A synthesis, Comptes Rendus Geoscience, Vol. 340, 761-770.

Douglas B.C. (1991) Global sea level rise, Journal of Geophysical Research, Vol. 96, No. C4, 6981-6992.

Iz H.B. (2006) How do unmodeled systematic mean sea level variations affect long-term sea level trend estimates from tide gauge data? Journal of Geodesy, Vol. 80, 40-46.

Jevrejeva S., Grinsted A., Moore J.C., Holgate S. (2006) Nonlinear trends and multiyear cycles in sea level records, Journal of Geophysical Research, Vol. 111, C09012, doi:10.1029/2005JC003229.

Katz E.J., Busalacchi A., Buschnell M., Gonzales F., Gourdeau L., McPhaden M., Picaut J. (1995) A comparison of coincidental time scales of the ocean surface height by satellite altimeter, mooring and inverted echo sounder, Journal of Geophysical Research, Vol. 100, No. C12, 25101-25108.

Kosek W. (2001) Long-term and short period global sea level changes from TOPEX/Poseidon altimetry, Artificial Satellites, Vol. 36, 71-84.

Lawrence S.P., Angell J.P. (2000) Evidence for Rossby Wave Control of Tropical Instability Waves in the Pacific Ocean, Geophysical Research Letters, Vol. 27, No. 15, 2257-2260. 
Legeckis R. (1977) Long waves in the eastern equatorial Pacific; A view from a geostationary satellite, Science, Vol. 197, 1177-1181.

Leuliette E.W., Nerem R.S., Mitchum G.T. (2004) Calibration of TOPEX/Poseidon and Jason altimeter data to construct a continuous record of mean sea level change, Marine Geodesy, Vol. 27, 79-94.

Liu W.T., Xie X., Polito P.S. Xie S.-P., Hashizume H. (2000) Atmospheric Manifestation of Tropical Instability Wave Observed by QuikSCAT and Tropical Rain Measuring Mission, Geophysical Research Letters, Vol. 27, No. 16, 2545-2548.

Miller L., Douglas B.C. (2004) Mass and volume contributions to twentieth-century global sea level rise, Nature, Vol. 428, 406-409.

Niedzielski T., Kosek W. (2005) Multivariate stochastic prediction of the global mean sea level anomalies based on TOPEX/Poseidon satellite altimetry, Artificial Satellites, Vol. 40, 185-198.

Niedzielski T., Kosek W. (2009) Forecasting sea level anomalies from TOPEX/Poseidon and Jason-1 satellite altimetry, Journal of Geodesy, Vol. 83, 469-476.

Niedzielski T. (2010) Non-linear sea level variations in the eastern tropical Pacific, Artificial Satellites, Vol. 45, 1-10.

Niedzielski T., Kosek W. (2010) El Niño's impact on the probability distribution of sea level anomaly fields, Polish Journal of Environmental Studies, Vol. 19, No. 3, 611-620.

Pezzi L., Caltabiano A., Challenor P. (2006) Satellite observations of the Pacific tropical instability wave characteristics and their interannual variability, International Journal of Remote Sensing, Vol. 27, No. 8, 1581-1599.

Philander S.G.H, Hurlin W.J., Pacanowski R.C. (1986) Properties of Long Equatorial Waves in Models of the Seasonal Cycle in the Tropical Atlantic and Pacific Oceans, Journal of Geophysical Research, Vol. 91, No. C12, 14207-14211.

Raudsepp U., Toompuu A., Kõuts T. (1999) A stochastic model for the sea level in the Estonian coastal area, Journal of Marine Systems, Vol. 22, 69-87.

Royston P. (1995) A Remark on Algorithm AS 181: The W Test for Normality, Applied Statistics, Vol. 44, 547-551.

Shapiro S.S., Wilk M.B. (1965) An analysis of variance test for normality (complete samples), Biometrika, Vol. 52, 591-611.

Wagner C.A., Tai C.K., Kuhn J.M. (1994) Improved ocean tide from TOPEX/POSEIDON and Geosat Altimetry, Journal of Geophysical Research, Vol. 99, No C12, 24853-24865.

Received: 2010-06-27,

Reviewed: 2010-09-01, by W. Popiński,

Accepted: 2010-09-10. 